\title{
The emotional dimension of life standard
}

\author{
Divisão de Psicologia do Hospital \\ das Clínicas da FMUSP
}

Since making origin, the study of life standard has been of great concern to scientists, however the expression "life standard" started to be used as of 1975 , according fo Sptizer.

In former studies the concern with life standard could be found in several ways since "state of health" was defined, in 1947, by the World Health Organization. It assimilated the health definition in the British Constitution which stated: "Health is not only the absence of infirmity and disease but also a state of physical, mental and social well-being".

Attempting to define, constitute and quality life standard, one could verify that not only health but also dicision may intervene in the union of body and mind, human relationships, identity, ability to create, decide and control of the individuals. When this stucture is jeopardized, the individual colapses, becoming alienated.

On the order hand, when including everything in the psychology domin one "evenctually creates a gap that renders false guarantees of control of experiences and facts, such as it occurs in a serious disease), where people have, in fact, little or no control at all".Sontag.

Between 1977 and 1987 a significant number of publications focused methods to evaluate life standard. In these studies as well as in later ones, this variable has been always associated with physic, phychic and social issues.

The approach to these studies had changed some what. In most cases, the questionnaires were developed in areas related to medical knowledge and recently they have been limited to diseases or even to specific differences of specialty, such as it occurs with oncology that refers in different way to breast, head or neck cancer. Estevez admitted other factors in addition to those abovementioned, such as the factor cost and benefit, not only the financial aspect of the treatment but also a possibility of a productive use of those materials for those who participate in the patient treatment process.

Creating methods to assess, in a scientific form, life standard has been pointed out in former studies, however there are still many controversies in the aspects "QUALITY" and Quantity.

In 1895, in one of his studies entituled "Project", Freud tried to study psychologic topics using a quantitative approach, and he stanted: "since recognizes only quantity, however we cannot use this notion in emotional dimension, wicvh can be observed not by the "quantity"in outer world but by the quality we achieve with the relations in outer world.

Estevez proved to be able to co-ordinate the life standard question including all its aspects, such as: life style, life goal and life reality.

We studied patients submitted either to vascular surgery or plastic surgery in the Hospital das Clinicas da FMUSP. In most cases, the studies were prospective, using similar appraisel instruments such as: 1 . specific questionnaire in semi-oriented interviews: 2. two reliable and sensible tests, the Human Figure Drawing (HFD) Machover, The CrownCrisp Experimental Index test (CCEI).

We tried not to outline personality aspects, but to observe proup characteristics.

In preliminary studies we observed that patients submitted to lower limb revascularization felt an opressive world, with very few perspectives and lack of confidence in social contacts, due to the permanent threat of the disease and the suffering of other patients. These patients experienced lossdue to constant physical changes, professional alterations (retirement) and the absence of a life project. In addition to those difficulties they seeked inner aids in oder to defend themselves such as: phobia, obsessiveness and hysteria.

In patients who had amputation at the thigh level, altough regression, attempt to refrain agressiveness, lack of confidence to interact in the social environment opressure feeling, which was demonstrated by physical signs more significant in depression and obsessiveness and phobia as a self defense.

By studying a new group we observed patients with intermittent claudication of lower limbs after one year of treatment. We evaluated aspects related to their life standard and their emotional dynamic, individual and characterization. In the first phase, the studies showed that the results were statistically significant applying the CCEI test: signs of phobia, hysteria and depression, Anxiety was the only significant trait in the female group. These results were confirmed by the HFD, 
that in addition, showed sexual impotence, lack of confidence in social contacts, introversion and defense by retraction, agressiveness that made them survive (which was also one of the characteristics of an hypertensive individual), lack of confidence when facing new situations, narcissism and egocentricity.

These aspects of life standard may also be studied in those who are not sick, such as the life standard of women who were submitted to rhytidoplasty. We verified that in this group there was a search to improve life standard through surgery. We understood their difficulties in the process of aging in which body schema did not correspond to their body image. Despite their social communicability, decision power adaptation and manipulation. They used an obsessive defense which allowed to control anxiety, depression and balance to face surgery. It reveals to be the measurer between body and mind, and the hysteria that increase their value in their female signs. In the second phase of the study, six months after the surgery, those same women were once again interviewed with results in the CCEI test tending considerably to anxiety, obsessiveness, depression and hysteria. Only due to this result could one state that there were no gains in the LS of it, but most of theses women referred to the advantages such as; feeling better with themselves, improvement in afecctive relationships, social climbing, improvement in professional life, among other aspects discussed.

In a new group of women who wanted to be submitted to reduction mastoplasty, the aspects related to life standard were also related to environment, with absence of confidence in social contacts associated with the sexuality problems. These women, specially because of their age, economic and professional position had stronger emotional support than those already mentioned. Six months after the surgery the most relevant factor we found was the improvement regarding depression, wich represented a considerable improvement in the life standard of this group (under study).

Studies about life standard are not only limited to translate changes regarding physical, psychologic and social aspects, but also to represent the possibility to improve the relationship between the doctor and his patient, which must not be viewed only as a disease carrier. 